\title{
Assessing the Performance Gap of Climate Change on Buildings Design Analytical Stages Using Future Weather Projections
}

\author{
Ibrahim ALHINDAWI ${ }^{1}$, Carlos JIMENEZ-BESCOS ${ }^{2 *}$ \\ ${ }^{1,2}$ Architecture and Built Environment, University of Nottingham, Nottingham, United Kingdom
}

\begin{abstract}
With the higher pace of climate change, temperatures are rising each year, resulting in various effects on the thermal status of buildings. This paper takes the opportunity of analysing different scenarios of greenhouse gas (GHG) emissions using hourly weather data of future projections by implementing EPW weather files on EnergyPlus software dynamic simulations, coupled with architectural science methods of climate analysis, to test the effect of high and medium-high emission scenarios for the 2050s and 2080s future timelines on thermal comfort range, passive zones potential, and heating/cooling periods, as compared to the weather data from 2003-2017. Simulations results have shown a remarkable effect on the scale of daily cooling hours and monthly coverage under the high GHG emission scenario, expanding its range by $60 \%$, with 6 hours on summer peak days and 3 months/year, as well as an annual decrease in heating period by $33.3 \%$. Thermal comfort zones of tested periods have also witnessed an alternation, translating the effect on the passive cooling and passive heating zones' way of variating, where the ranges are pushed towards their potential limits. Results have also demonstrated that if future weather data is not included in simulations, a weather-related performance gap is generated.
\end{abstract}

Keywords - Cooling-heating period; future weather; psychrometry; thermal comfort

\section{INTRODUCTION}

The impact of climate change on the building sector has been under the focus of many researchers world widely, while the energy consumption and thermal status of building designs were the main aspects of investigations and studies. The building industry approach to researching the impact of climate change, usually focus on energy demands of buildings, and in order to assess the climate change circumstances in the future, weather data projections are needed to simulate the building performance in the future.

Different methods for future weather projections exist, in the shape of mathematical approaches, with a variety of formats, scenarios of emissions and tools of prediction that represent simulation of weather data, depending on the region of simulation package. In the city of Zurich-Kloten in Switzerland, Frank T. H. [1] has conducted a similar approach of this paper by investigating multi-story buildings of different uses for the change of energy demand, projecting hourly weather data of a historical range from 1981 to 2003 according to four future emissions scenarios:

- Scenario A: WMO (World Meteorological Organisation) [2] normal;

* Corresponding author.

E-mail address: carlos.jimenez-bescos@nottingham.ac.uk 
- Scenario B: IEA Design Reference Year;

- Scenario C: Average reference year;

- Scenario D: Warm reference year, specifying increases in cooling demands, and change in loads calculated in terms of historical-future comparisons.

Another contribution to future building energy demand studies in Europe by Olonschech et al. [3] have focused on the climate change impact on the energy consumption in the German housing sector, using statistical analysis on HDD (heating degree days), and CDD (cooling degree days) as well, under the scenario of A1B of the Intergovernmental Panel on Climate Change (IPCC) [4] greenhouse gas (GHG) emission, while Chow et al. [5] has worked on developing another method of future weather data file, the Q-Sin method in the UK, in contrast to the method used in this paper. Relevantly to this research's assessment, Belcher et al. [6] have worked on producing design weather data for building future weather simulations by developing a morphing method, a well-known method for future weather generation.

Other tools for the future weather projections have been under development as well, as Jentsch et al. [7], [8] have developed the CCWorldWeatherGen tool, by the implementation of Microsoft Excel, allowing users to generate weather data using EnergyPlus EPW file formats, similar to what is partially approached in this study, and the Typical Meteorological Year (TMY2), where they have also used the morphing method in their study as the future weather projection algorithm, using the IPCC GHG emission A2 scenario of medium-high intensity into file formats of EPW and TMY2 files, while the current study investigates the A1FI scenario as well. Another use of the CCWorldWeatherGen tool has been recorded for Sabunas et al. [9] where he generated future weather data for evaluating energy consumption in buildings for the 2020s, 2050s and 2080s periods. Moreover, these timelines are tested in this paper's simulations as well, disregarding the 2020 s considering it very close to the $2003-$ 2017 range already simulated for comparison.

Use of future weather data projections for different research investigations have been also conducted in other regions of the world, as in Japan, where Arima et al. [10] have applied a study method of dynamical downscaling on residential buildings, using the scenario of MIROC4h of predicting future local weather, while Kikumoto et al. [11] also used future weather projection to assess climate change impact on buildings in the future.

The morphing method has been used in China as well, where the development of a futureprojected hourly weather files has been implemented by Wan et al. [12] to produce different parameters, as temperatures of dry and wet bulb, rainfall levels predictions, and solar radiation intensities, as the test has been applied by simulating different types of building uses in Hong Kong, where a similar GHG emissions levels reference to this paper's, the scenarios of IPCC has been tested. Using the morphing method, composite GHG emission scenarios have been also integrated in generating future TMYs by Zhu et al. [13] in Shanghai, for prototypical building models. The scenarios tested were RCP4.5's of S1, S2 and S3, baselined on IWEC-version TMY condition, which is a varied set of scenarios than the ones used in this paper. A project by Huang et al. [14] projected the monthly data of future weather of World Climate Research Programme (WCRP) [15] Phase 3 (CMIP3) of inter-comparison coupled model, where in their study, they have generated monthly future hourly weather conditions for each of the test locations, conducting an investigation using EnergyPlus, looking into the relationship between building energy and climate change, with a variety of building types and scales of spatiotemporal, using a transient-state approach of simulations as this paper's assessment does. Where also relevant to this paper's way of investigation, another application of the morphing method, by Wang et al. [16], for the purpose of 
generating future weather files, implementing the A2 scenario of HadCM3 for the periods of 2020s, 2050s and 2080s, varying from this paper's approach by using the periods of 2020 2089 from NCAR Community Earth System Model version 1 (CESM1) for RCP2.6, RCP4.5 and RCP8.0 scenarios. In their study, they have investigated an office building, by simulating energy use and sustainable design exploration with EnergyPlus software.

Adding to the topic of GHG emission scenarios discussion, Jianga et al. [17] in University of North Florida [18] have discussed GHG emission scenario sources and types, for instance, the Swiss Federal Office of Meteorology and Climatology creates the CH2018 Climate Change Scenarios [19]. Japan has also developed MIROC4h as Takashi et al. [20] mentioned.

Quoting Wang et al. [16], discussing the HadCM3 model which is a reference of this study's approach; "Among these GCMs, HadCM3 is the most well-known developed by the Hadley Centre in the United Kingdom. Like other GCMs, it is a grid point model in which the spatial resolution of each grid cell is $2.5^{\circ}$ in latitude and $3.75^{\circ}$ in longitude. In the meantime, the model provides the monthly change in dry-bulb temperature, diurnal temperature variation, relative humidity, wind speed, and solar radiation, which have a major impact on the building heating and cooling load and can be found on Intergovernmental Panel on Climate Change (IPCC) website [21]".

In a study focusing on the impact of user behaviour and how to incorporate user behaviour on building simulation, Jimenez-Bescos et al. [22] have noticed a reduction in comfort due to future weather will generate a change in behaviour from users to adapt to higher temperatures.

Albatayneh et al. [23] have also investigated the significance of building design for the climate from the point of view of how important is the climate conditions for building design and the effect on the design and sustainability on energy savings due to changing climate zones, in a way to testing for future climate, is to move the buildings into other climate zones.

In another paper incorporating future weather conditions, Rucevskissandris et al. [24] have researched the optimisation of PCM parameters and acknowledge the influence of climate on the parameters selection, which will be affected by future weather with the PCM not able to perform as expected if higher temperatures for night ventilation reduce passive cooling.

Throughout the wide variety of future weather projection methods, implemented energy simulation software (e.g. TRNSYS, EnergyPlus, Visual DOE), it is quite clear that the morphing method is the algorithm that is mostly used to generate future weather projections. Multiple emission scenarios have been implemented as well, however, the IPCC GHG emission scenarios are the ones mostly used among the different projection methods approaches, therefore, it has been implemented in achieving this study's aim, which comprises conducting an analytical approach to assess the impact of climate change in the future on building pre-design analysis and early design stages. Furthermore, the future timelines thermal status effects on the building design approaches and stages of decision making was investigated, including the calculations of different aspects of thermal characteristics that set boundaries to assess whether the building design simulations results are adequate for closing the future performance gap.

\section{METHODOLOGY}

The methodology of this assessment has been built with a four steps approach, represented by the implementation of dynamic simulations through EnergyPlus software, where the 20032017 EPW base weather files for a test location in Chelmsford, England, have been uploaded 
to the software, taken as a base reference source for the most recent range of weather data records.

For generating future weather data, the 2003-2017 EPW was uploaded on Weather Morph: Climate Change Weather Data Generator online platform with HadCM3 scenarios [17], [18]. Based on IPCC assigned GHG emission scenarios, according to technology development, social trends, demographic factors of population and economic growth and related description [4]. Weather Morph takes grid points in the time slices of 2020, 2050, and 2080, represented by the monthly changes of weather variables in the generated EPW files used in the simulations. Description of these scenarios was categorised into four levels, simulating the future GHG emission:

Scenarios: Low (B1), medium-low (B2), medium-high (A2), High (A1FI).

Three Timelines: 2020s, 2050s and 2080s.

The first step is to simulate the base weather file of 2003-2017 data on the Weather Morph platform and generate the EPWs of 2050s and 2080s timelines through different scenarios. The chosen scenarios of simulation for this study are A2 and the A1FI. Additionally, three main parameters are extracted from the weather files and scenarios of test, 2003-2017 (historical base), 2050-A2, 2050-A1FI, 2080-A2, 2080-A1FI for the implementation in the second step; dry bulb (DB) temperature, wet bulb (WB) temperature and relative humidity (RH), all on an hourly basis for every day of the year (365 days x 24 hours).

Step 2 includes filtering and scheduling of the specifically needed sets of the extracted parameters in the previous step, where each day's hourly data of the parameters are examined for maximum and minimum recorded values through 24 hours of each day of every month, ending up with maximum-minimum DB, maximum-minimum WB and maximum-minimum $\mathrm{RH}$ produced by simulations of each timeline and scenario mentioned in the first step.

The next two steps of this study are realised through an architectural science method of climate analysis, derived from two main sources of sustainable building design related climate analysis through architectural pre-design processes; Szokolay [25], where the process of integrating the physics of heat and temperature, psychrometry and thermal comfort are merged into a combination of a stepped analysis, producing multiple outcomes, and Olgyay [26], as it sources the perspective of the bioclimatic approach, comfort zone, regional evaluation and climatic elements of weather factors and heat characteristics.

Step 3 comprises calculating the effective temperature in its maximum and minimum values, to produce the hourly effective temperatures table for the purpose of charting the cooling and heating periods for the 2050s-A2, 2050s-A1FI, 2080s-A2, 2080s-A1FI as well as the base weather set of 2003-2017 as a comparison reference. As Ogunsote et al. [27] mentioned, The Effective Temperature (ET) is defined as the temperature of an atmosphere where it is saturated and still, which produces the same effect in the absence of radiation. Combined effects are indicated by the ET, including temperature and clothing, relative humidity, and air velocity. Choudhury et al. [28] concludes, about the ET, that it cannot be measured by a thermometer, as it is determined experimentally on its index by combining parameters like DB, WB, RH, radiant conditions (MRT) and air movement, inducing the same thermal sensation. The combinations of DB and WB gives produces the new ET of a given space, of a thermo-equivalent environment at $50 \% \mathrm{RH}$, inducing the same sensation of cold and warmth.

The method used in this step to acquire ET sets is processed through the "Nomogram for the Effective Temperature index", as the extracted parameters of maximum and minimum sets of DB-WB produces maximum and minimum ET. Indicating most of the effects on comfort's major factors is a merit the index offers, besides being easy to use and interact. 
Furthermore, hourly charting of maximum and minimum ET, produces the hourly ET temperatures tabulation process for the cooling and heating periods. The mentioned process is done through all test timelines and emission scenarios mentioned earlier. Fig. 1 shows the max/min ET charting example.

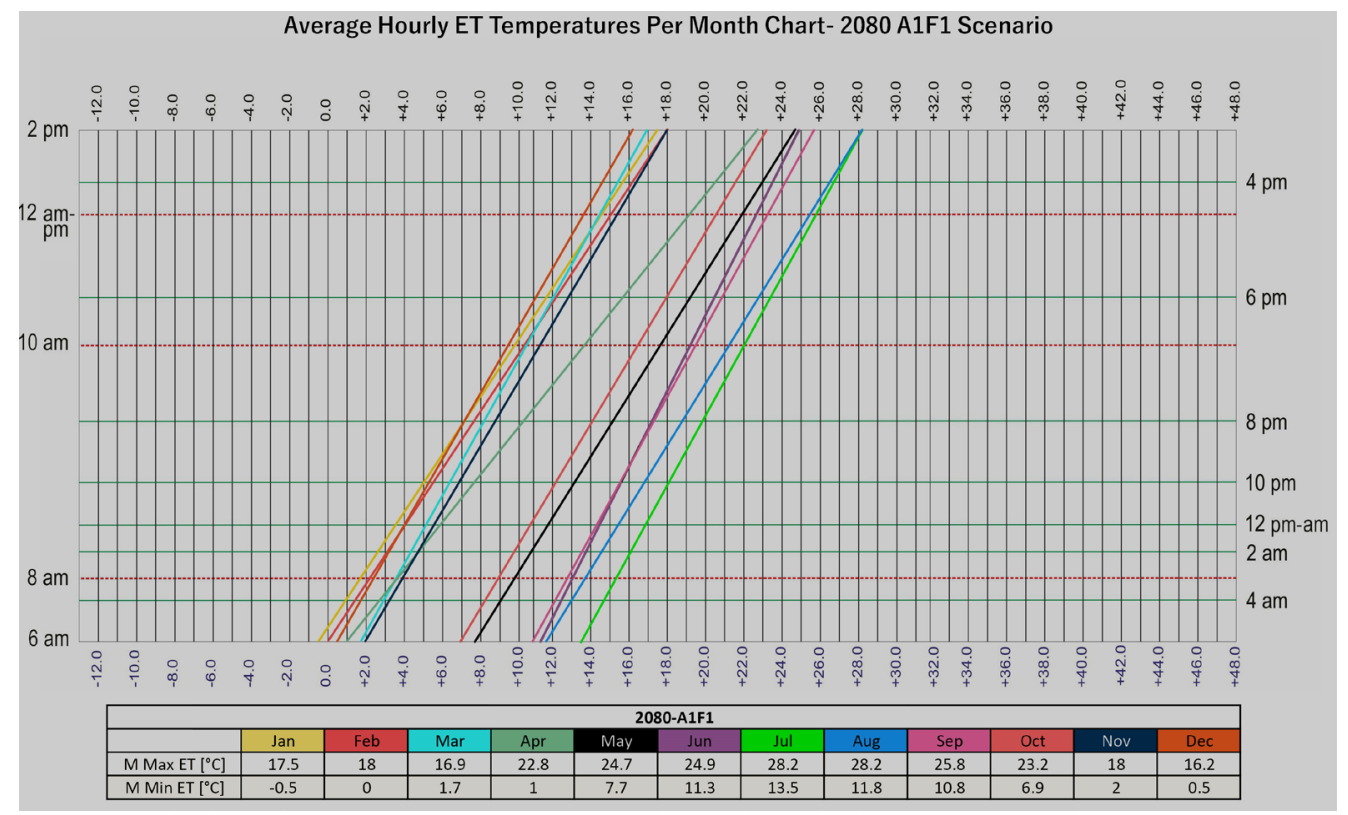

Fig. 1. Hourly ET Production Process Example - 2080 A1FI.

Step 4 was conducted for the purpose of producing psychrometric charts for each EPW weather file, showing three outputs; thermal comfort zone, passive cooling zone (natural ventilation potential) and passive solar heating zone, in order to acquire a comprehensive image of what these elements would look like, perform and interact through the different circumstances of climate change projected future weather parameters. The method takes the thermal comfort of balance, factors, adjustment mechanisms and comfort indices as a theoretical background to reach comfort zone limits of range determination and placement on the psychrometric charts, and its relation to the passive cooling and passive solar heating zones, specifying all zones' limits, potentials and variations through different weather scenarios and climate change impact implications.

The process implements a correlated sequence of equations, shown in Fig. 2, leading to the specification of the boundaries that set limits of the mentioned zones above, depending on a list of parameters that might change by value according to location, weather conditions and regional climate zones. However, these parameters have been taken as values that are fixed for all the simulations as they are not the main aspects of this study, for the reasons of comparisons. 


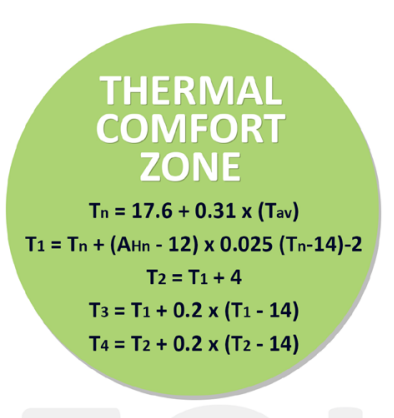

Tav: Average Annual Temperature fir the Site, ${ }^{\circ} \mathrm{C}$. Hv: Mean Daily Total Solar Radiation Intensity on the Equator Facing Vertical Surface, $W / \mathrm{m}^{2}$. ( $\left.\mathrm{H}_{v}=100\right)$

Eff:: Efficiency of the Passive Solar Heating System, Dimensionless. (0.5)

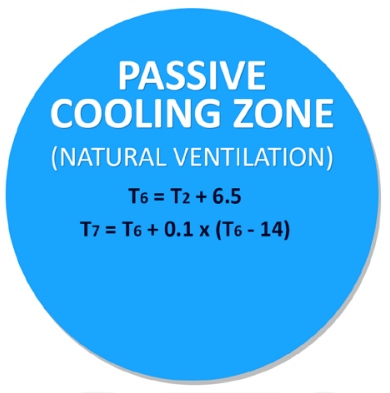

Tn: Neutral Temperature, ${ }^{\circ} \mathrm{C}$. Asg: Area of the Equator Facing Vertical Glazing, m2. $\left(A_{s g}=4\right)$ $U$ : Average U-value of the Building, $W / m^{2} .^{\circ} \mathrm{C} .(U=1)$

Fig. 2. Psychrometry Equations.

Fig. 3 diagram describes the detailed workflow of the methodology.

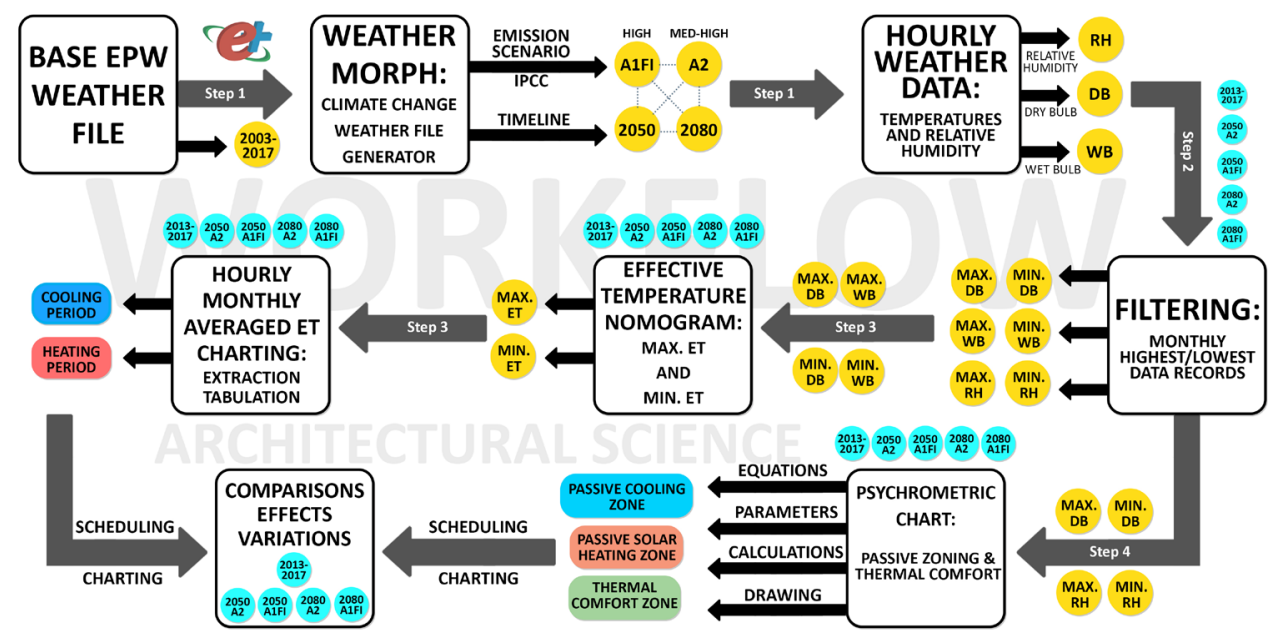

Fig. 3. Methodological Workflow Diagram.

\section{Results}

Recapitulating the aim of this research, as it rotates around the focus of acquiring an understanding of the thermal periods specifications of the building environment during the design stages for multiple climate conditions, the results are categorised into three sections; cooling periods, heating periods and psychrometry which is sub-categorised into thermal comfort zoning, passive cooling zoning and passive solar heating zoning. 


\subsection{Cooling Periods}

The results of the five tested weather files have varied in range, taking the $20-30{ }^{\circ} \mathrm{C}$ range of temperature as a reference, where cooling is needed, graduating from natural ventilation up to active methods where temperatures are above the thermal comfort zone. The 2003-2017 (historical) simulation have recorded a $23.1{ }^{\circ} \mathrm{C}$ maximum monthly-averaged hourly ET temperature in July and $-4.6^{\circ} \mathrm{C}$ in January, reflecting on the cooling period's pattern by a monthly range that starts in early April with less than one hour of cooling demand, which expands until it reaches its peak in July with just above 5 hours, then starts decreasing until reaching an end in early September days with around 2 hours.

As in Fig. 4, in the 2050s timeline of the A2 scenario (medium-high), a maximum monthlyaveraged hourly ET of $25.6^{\circ} \mathrm{C}$ was recorded in July and August, opposed by a minimum of $-2.7^{\circ} \mathrm{C}$ in January, translated into the pattern of cooling period that starts by the beginning of April with just under 4 hours of cooling demand in a mid-April day, peaking in a July day with more than 8 hours, recording an end of the cooling period by the beginning of October with 4 hours of cooling demand in a mid-September day.

For the A1FI-2050s timeline, a peak monthly-averaged hourly ET of $26.4{ }^{\circ} \mathrm{C}$ have taken place in August, and a minimum of $-1.4{ }^{\circ} \mathrm{C}$ in January. The cooling period pattern has marked a start in late March days with an hour of cooling demand, and under 4 hours in a mid-April peak day.

The number of hours reaches 9 in a peak day in July to mid-September that records 5 hours of cooling demand, ending in October by the end of the first $37 \%$ of its days.

Going through the 2080 s timeline, the A2 scenario witnesses a maximum monthly-averaged hourly ET of $27.1^{\circ} \mathrm{C}$, recorded in August, and an ET minimum of $-1.5^{\circ} \mathrm{C}$ in January. Cooling demand range starts by the start of the last 10 days of March, with 3 hours at its maximum, and 7 hours in May's maximum. The peak of cooling demand hours has made a statement in a July day, with just under 10 hours of cooling demand, heading to September' maximum and recording 5 hours, while reaching the end of the cooling period by the end of October's first $33 \%$ of its days.

The last case of cooling periods, belonging to the 2080s timeline of high GHG emission simulation (A1FI), recording a monthly-averaged hourly ET peak of $28.2^{\circ} \mathrm{C}$ in August, and a monthly-averaged hourly minimum ET of $-0.5{ }^{\circ} \mathrm{C}$, drawing a pattern that marks a cooling period start by mid-March, with more than 3 hours by the end of it. More than 8 hours in a peak day of May, takes the increase to a peak in July with just under 11 hours in the first two days, while 7 hours in a peak September day take the period through to its end by the end of the first half of October which records 4 hours of cooling demand as a peak.

\subsection{Heating Periods}

The range of heating demand ideally goes from $19-20^{\circ} \mathrm{C}$ down, where temperature drops below the thermal comfort zone, starting from below $0{ }^{\circ} \mathrm{C}$ temperatures to $0{ }^{\circ} \mathrm{C}, 0-10{ }^{\circ} \mathrm{C}$ and $10-20^{\circ} \mathrm{C}$ ranges. The heating periods of different scenarios are presented for the ranges between -10 and $20^{\circ} \mathrm{C}$, as the characteristics of a heating demand-dominance climate is obvious from the pattern in Fig. 5. The reference outline of effect for the cold period is shrinking by the effect of the climate change and taken from the very cold range of below $0{ }^{\circ} \mathrm{C}$ to $10{ }^{\circ} \mathrm{C}$ ranges, where heating is needed and met by passive and active methods during this period.

For the 2003-2017 timeline, the peak of heating daily hours reached 22 hours in a January day, where the heating demand technically starts in August for few hours in the very early morning, however, it reaches 6 hours in September as a kick-off to the demand records, 
decreased towards May with an end mark before June with around 2 hours in the very early morning.

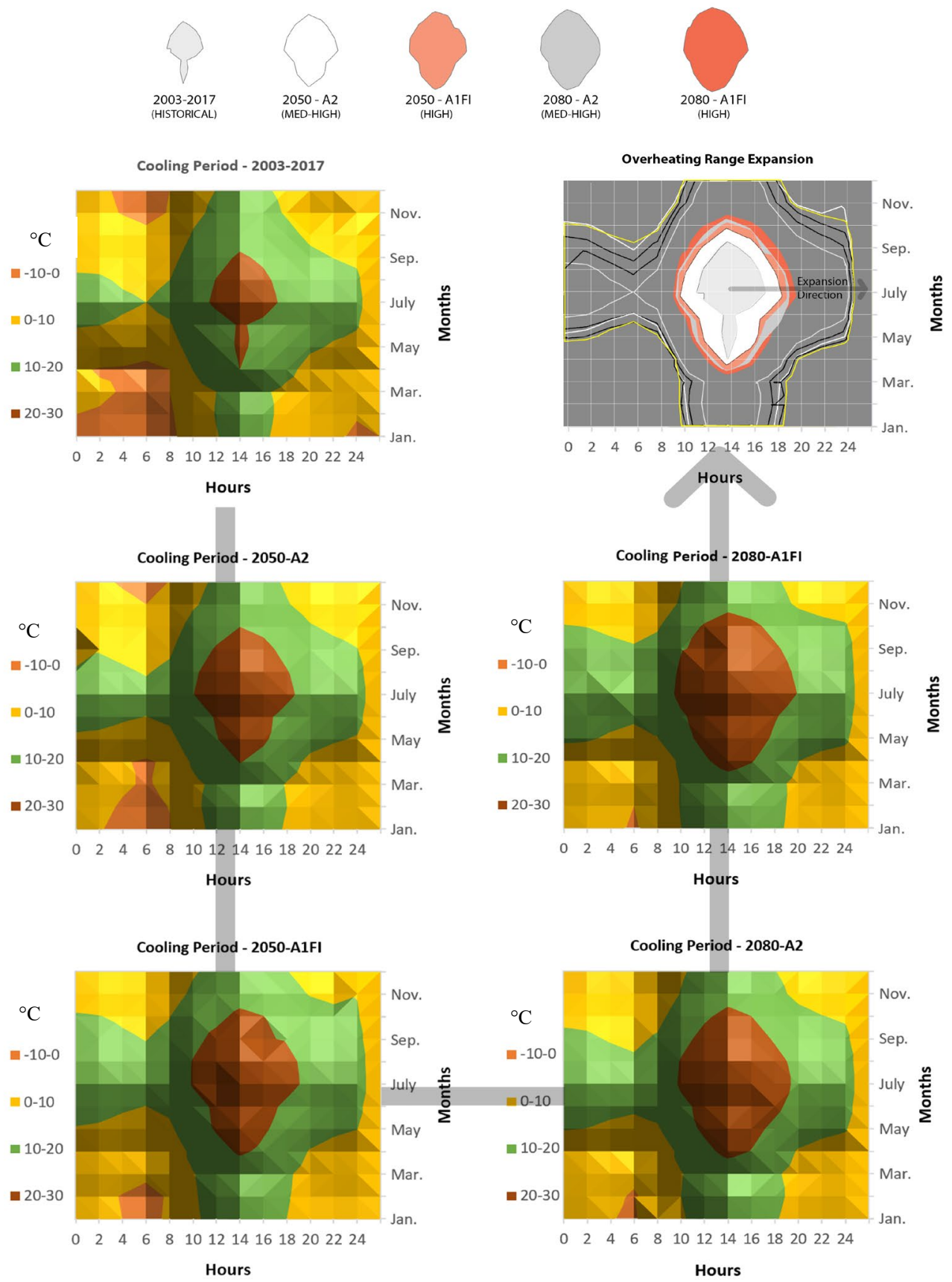

Fig. 4. Cooling Periods Sets and Comparisons. 
The 2050s A2 scenario marked a slight start by the end of August, with 2-3 hours only between 4:00-6:00 am, peaking in December with 17 hours, continuing at the same pace until March, and starts decreasing towards mid-May with 2 hours in a maximum limit daily of demand.

In the 2050s' A1FI scenario of high emission, mid-September day of peak heating demand marks a start of the period with 2 hours of demand recorded between 4:00-6:00 am, as Fig. 5 shows. Peak day of heating demand hits within the last third of December ending, recording 16 hours, keeping around this pace with decreasing towards the beginning of March, where it starts lowering gradually until ending in the first third of May with 3 hours of heating demand.

Traveling further in time in Fig. 5, the 2080s A2 scenario has a pattern of heating period that sets a starting point by the start of October, and ending by the end of April, recording a peak in the last two days of November and the first 3 days of December, with 14-15 hours of heating demand.

While October records 10 hours, the heating demand in February of this scenario records 13 hours as a representation of the diminishing numbers direction of the heating period towards the range's limits. For the 2080s, the A1FI scenario draws a pattern of heating demand that records a start in mid-September, and an end in early May, both with the fewest hours of heating demand with 3-4 hours during the maximum days. The peak of heating demand hours is set during some days of late November and early December, with around 15 hours a day, where the pace is similar to November to April, and clearly lower from November down to September.

\subsection{Psychrometry}

Psychrometry charts are categorised into thermal comfort zones, passive cooling zones (natural ventilation potential limits) and passive solar heating zones as represented in Fig. 6.

\subsubsection{Thermal Comfort Zones}

From $18.5-24.2{ }^{\circ} \mathrm{C}$, the psychrometric chart has translated the 2003-2017 EPW data into a thermal comfort zone, set as an outline as shown in Fig. 6. In the 2050s-A2 simulation, the thermal comfort zone's bottom limit draws a line at the temperature of $19.1^{\circ} \mathrm{C}$, ranging towards $24.9^{\circ} \mathrm{C}$. The range of the A1FI-2050s timeline has recorded a bottom limit of $19.6^{\circ} \mathrm{C}$, extending to the top limit until reaching $25.5^{\circ} \mathrm{C}$.

The A2-2080s simulation has illustrated the same range of thermal comfort zone on the psychrometric chart, starting from $19.6^{\circ} \mathrm{C}$ and ending at $25.5^{\circ} \mathrm{C}$ as well. Shifting up again, the 2080s' A1FI scenario has set the boundaries of the thermal comfort zone at the temperatures of $20-25.9^{\circ} \mathrm{C}$. Fig. 6 clarifies the mentioned ranges in a sequenced psychrometric charts order.

\subsubsection{Passive Cooling Zones}

These zones, as rendered in Fig. 6, are a depiction of the weather data of the test timelines on the psychrometric chart. The temperature (T6) represents the top limit of each passive cooling zone's potential, where the indoor temperature can be brought down to comfort zone from.

The 2003-2017 data sets a passive cooling potential limit of $29^{\circ} \mathrm{C}$, where natural ventilation could bring the temperature down to the comfort zone of $18.5-24.2{ }^{\circ} \mathrm{C}$, achieving a potential of $4.8+{ }^{\circ} \mathrm{C}$ passive cooling, depending on the indoor circumstances. 

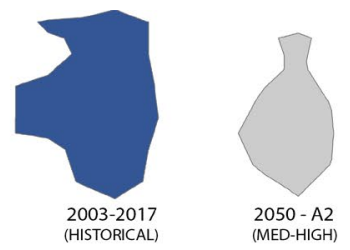

Heating period 2003-2017
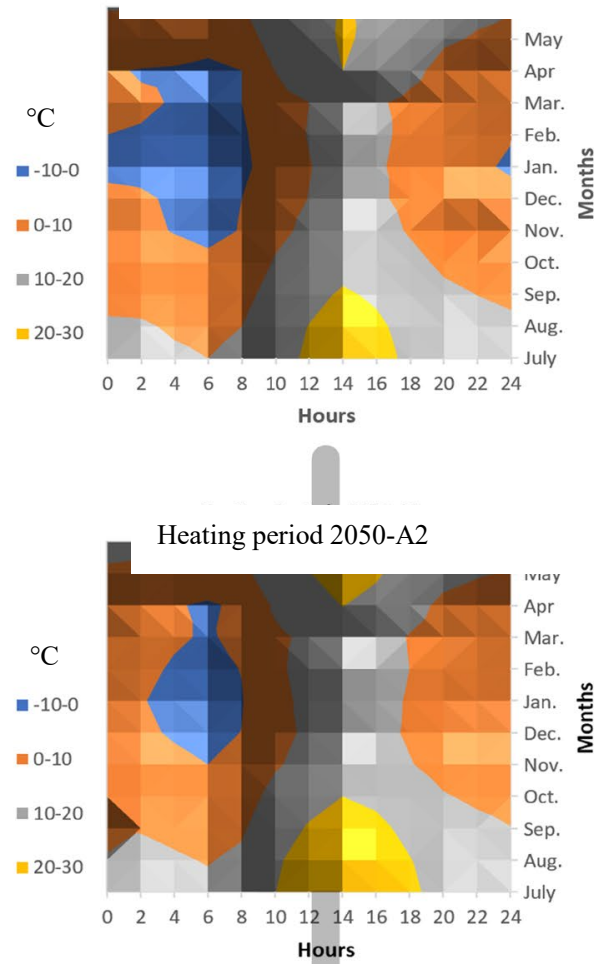

Heating Period - 2050-A1FI

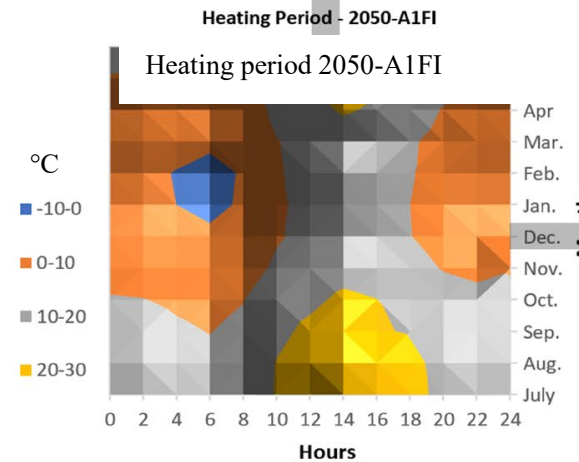
(n)

.

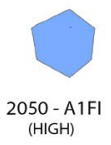

\section{$\sum^{\frac{n}{2}}$}
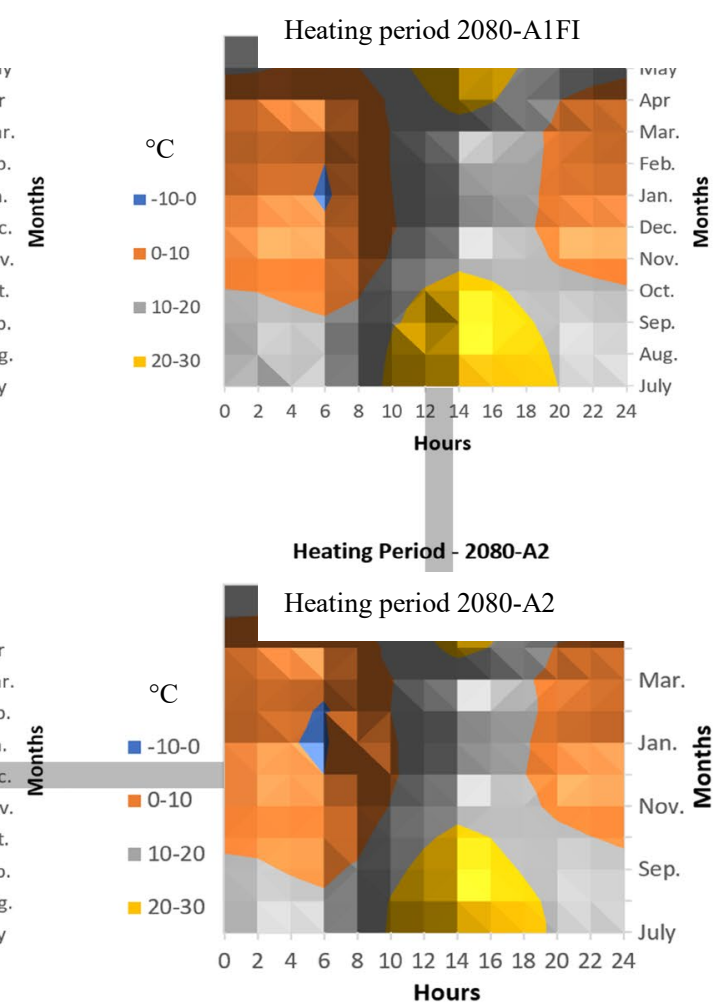

Fig. 5. Heating Periods Sets and Comparisons. 
A natural ventilation potential of $4.7^{\circ} \mathrm{C}$ has been calculated on the $2050 \mathrm{~s}-\mathrm{A} 2$ psychrometric chart, where the temperature can be brought down to a thermal comfort zone of $19.1-24.9{ }^{\circ} \mathrm{C}$, by a $29.6{ }^{\circ} \mathrm{C}$ passive cooling zone top limit.

For the 2050s-A1FI simulation, the passive cooling zone limit was found equal to $30.1{ }^{\circ} \mathrm{C}$, meaning that the natural ventilation potential is predicted to be $4.7+{ }^{\circ} \mathrm{C}$, bringing down the indoor temperature to the comfort zone top limit of $25.5^{\circ} \mathrm{C}$.

From the 2080s-A2 simulation, the potential of natural ventilation had an outcome similar to the 2050s-A1FI results, as both thermal ranges hoover on the same levels. Unlike the previous scenario, the $2080 \mathrm{~s}-\mathrm{A} 1 \mathrm{FI}$ simulation had a passive cooling zone limit of $30.5^{\circ} \mathrm{C}$ that produces a potential of $4.6+{ }^{\circ} \mathrm{C}$, from $30.5^{\circ} \mathrm{C}$ down to the thermal comfort zone top limit of $25.9{ }^{\circ} \mathrm{C}$.

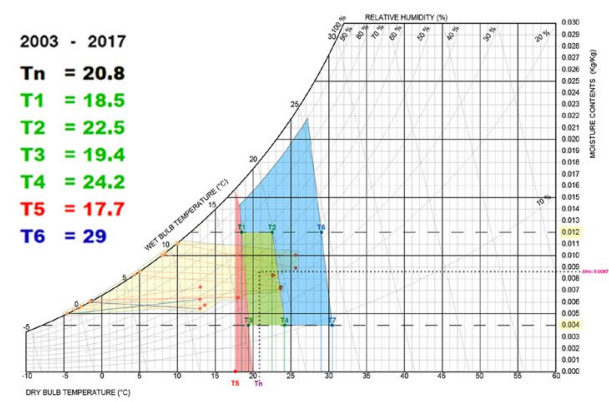

2003-2017

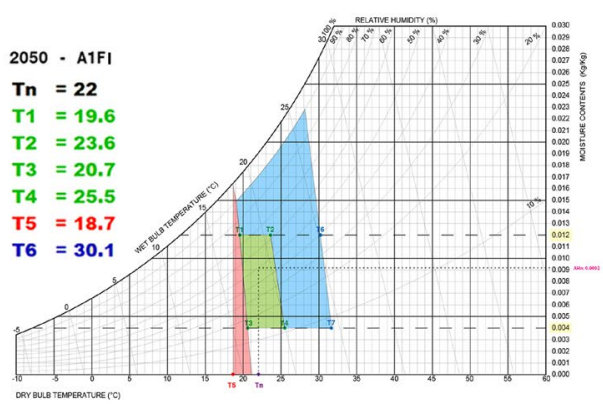

$2050 \mathrm{~A} 1 \mathrm{FI}$

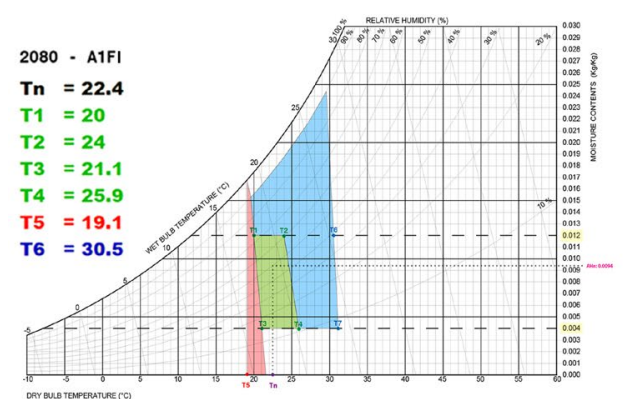

$2080 \mathrm{~A} 1 \mathrm{FI}$

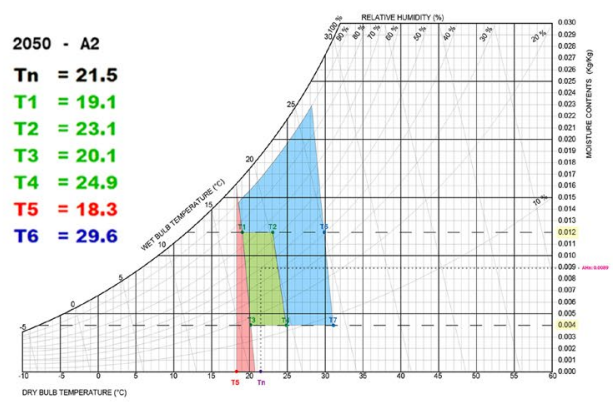

2050 A2

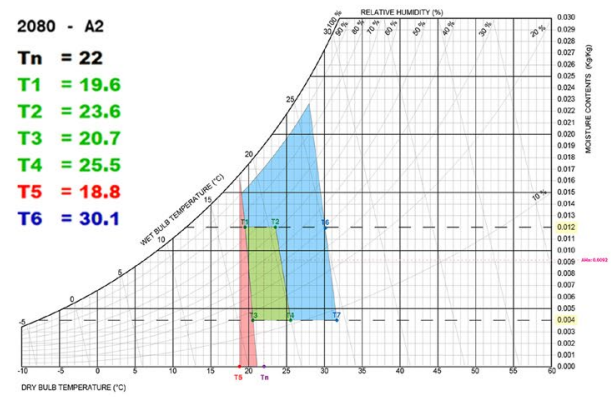

2080 A2

Fig. 6. Psychrometric Charts - Zones Comparisons. 


\subsubsection{Passive Solar Heating Zones}

Passive heating zones rendered on the psychrometric charts earlier in Fig. 6 are based on T5 from the equations in Fig. 2, as T5 represents the temperature that solar heating starts being effective by, pushing the indoor temperatures from T5 value up to the thermal comfort zone range.

Results of passive solar heating ranges were as follows:

- 2003-2017:

$\mathrm{T} 5=17.7^{\circ} \mathrm{C}$, Potential $=0.8+{ }^{\circ} \mathrm{C}$

- 2050 A2:

$\mathrm{T} 5=18.3^{\circ} \mathrm{C}$, Potential $=0.8+{ }^{\circ} \mathrm{C}$

- 2050 A1FI:

$\mathrm{T} 5=18.7^{\circ} \mathrm{C}$, Potential $=0.9+{ }^{\circ} \mathrm{C}$

- 2080 A2:

$\mathrm{T} 5=18.8^{\circ} \mathrm{C}$, Potential $=0.8+{ }^{\circ} \mathrm{C}$

- 2080 A1FI:

$\mathrm{T} 5=19.1{ }^{\circ} \mathrm{C}$, Potential $=0.9+{ }^{\circ} \mathrm{C}$

Fig. 7 illustrates each passive solar heating base limit result for each of the scenarios of the study, where the transparent red-coloured range represents the potential of the passive solar heating with respect to the thermal comfort zone.

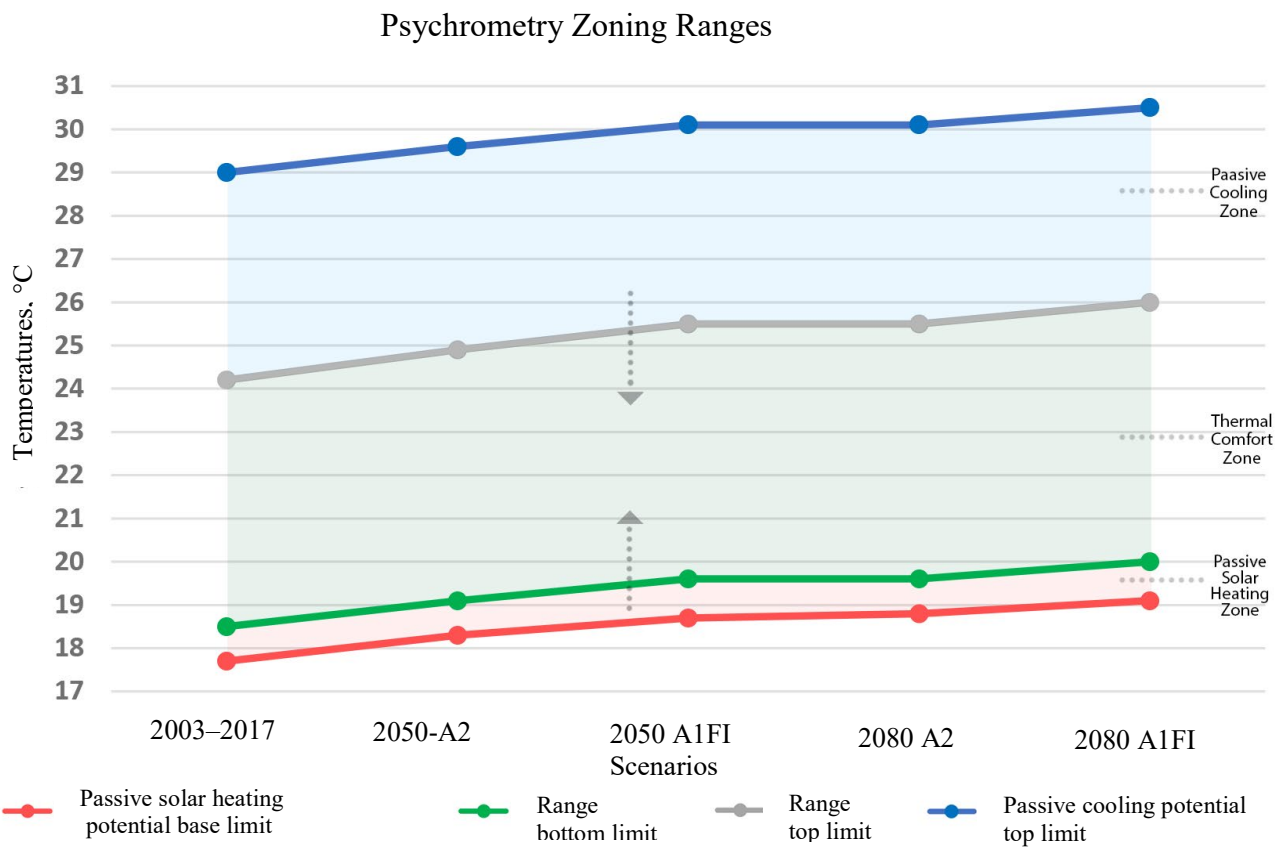

Fig. 7. Psychrometry Zoning.

\section{DiscuSSION}

\subsection{Cooling Periods}

The ranges of effective temperatures have grown larger in scale through the increasing GHG emission scenarios sequence. observing the green gradient $\left(10-20^{\circ} \mathrm{C}\right)$ form previously shown Fig. 4, through the different scenarios' patterns, a very notable increase in the temperate levels of heat range throughout the escalating GHG emission projections has been noticed. 
The issue lies in the $20-30{ }^{\circ} \mathrm{C}$ gradient, where the cooling demand hours have dramatically increased with the higher $\mathrm{GHG}$ emission scenarios, resulting in an increase of overheating hours.

Furthermore, the 2003-2017 to the 2080s-A1FI iterations showed an increase, not only in the cooling demand hours, but in the annual range of cooling period, where it expands from starting narrowly in April and ending in late August for the 2003-2017 timeline data, to starting in mid-March and ending in mid-October during the highest emission scenario, with double the daily hours of cooling demand, and double the annual peak days in July and August. As an interpretation to the noticed results, a cooling demand peak day in July from the 2003-2017 timeline weather data recorded 5 hours of cooling demand, yet, going through the higher emission scenarios, specifically the A1FI of 2050s and 2080s, peak days in July with 9 and 11 hours of cooling demand have been recorded respectively. Fig. 8 shows the comparison between each scenario and timeline peak cooling demand daily hours and annual range, where the climate change strong effect is predicted to more than double the effect.
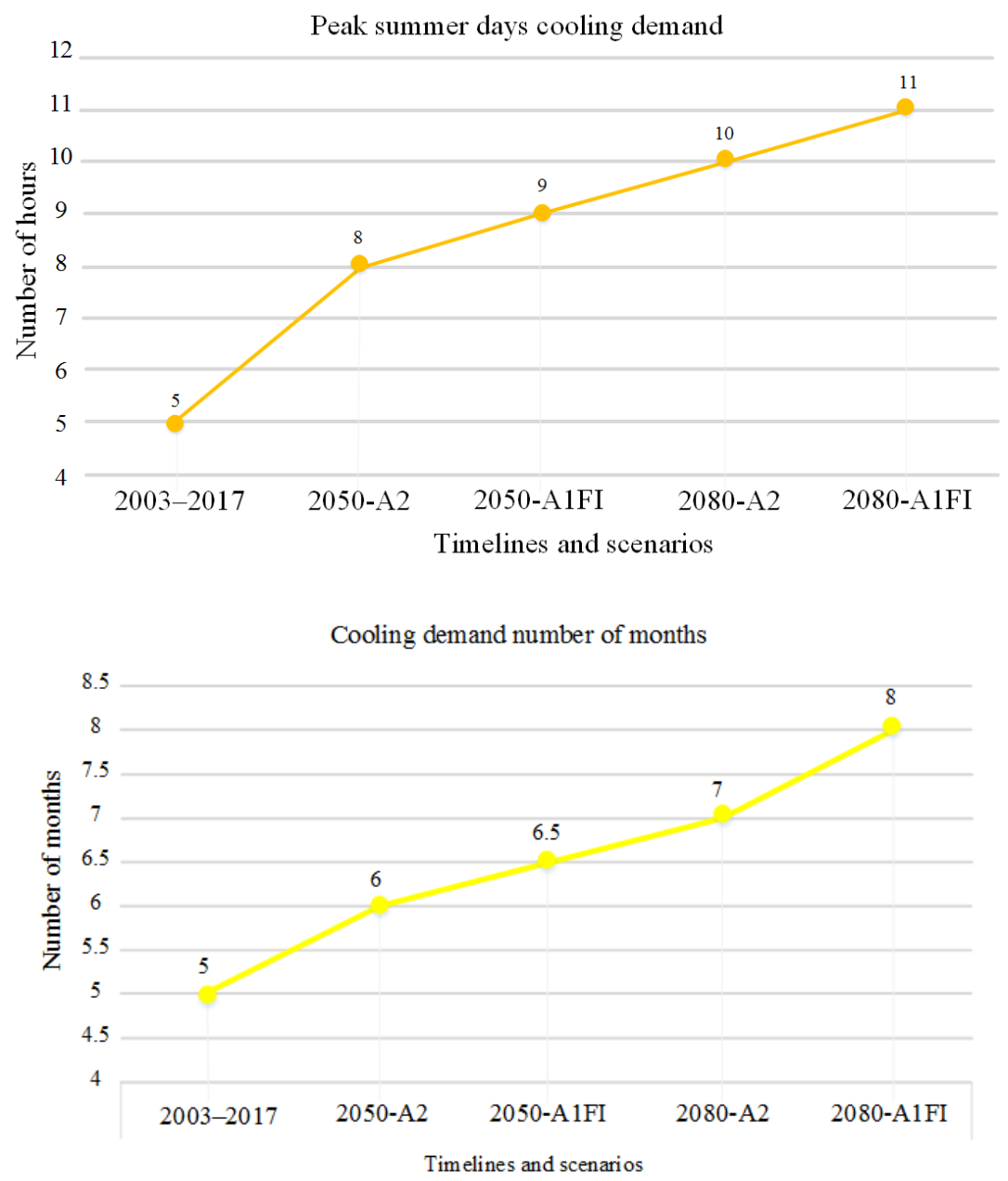

Fig. 8. Cooling Period Specific Comparison. 


\subsection{Heating Periods}

As a result of higher temperatures due to higher GHG emission scenarios, heating demand have been reduced throughout the escalating sequence of the studied timelines.

The severe effect of climate change on the temperatures is predicted to cause a process that holds large similarity to the climate change melting effect on the North Pole throughout the last century, as presented by NASA historical observations [29]. By metaphor, this effect is clearly shown while examining the blue gradient of minus temperatures to $0{ }^{\circ} \mathrm{C}$ in Fig. 5 as a shape that is melting like ice throughout the escalating GHG emission scenarios, where this range gradually vanishes from the months of October, November, February, March and even April in 2003-2017 timeline to only mid-December up to the end of January in 2080s A1FI GHG emission scenario.

The reduction in heating demand hours during the heating period months has been noticed. A heating demand peak of 22 hours has been recorded in January, which was dramatically reduced if compared to a peak day in the 2080s A1F's January peak day, where it is 7 hours less in demand, making it 15 hours only during that peak day, which is an extreme effect in a location in the UK for the coldest day in a year. The gradual reduction effect of the escalating GHG emission pace could be noticed in Fig. 9, where all scenarios and timelines are compared.
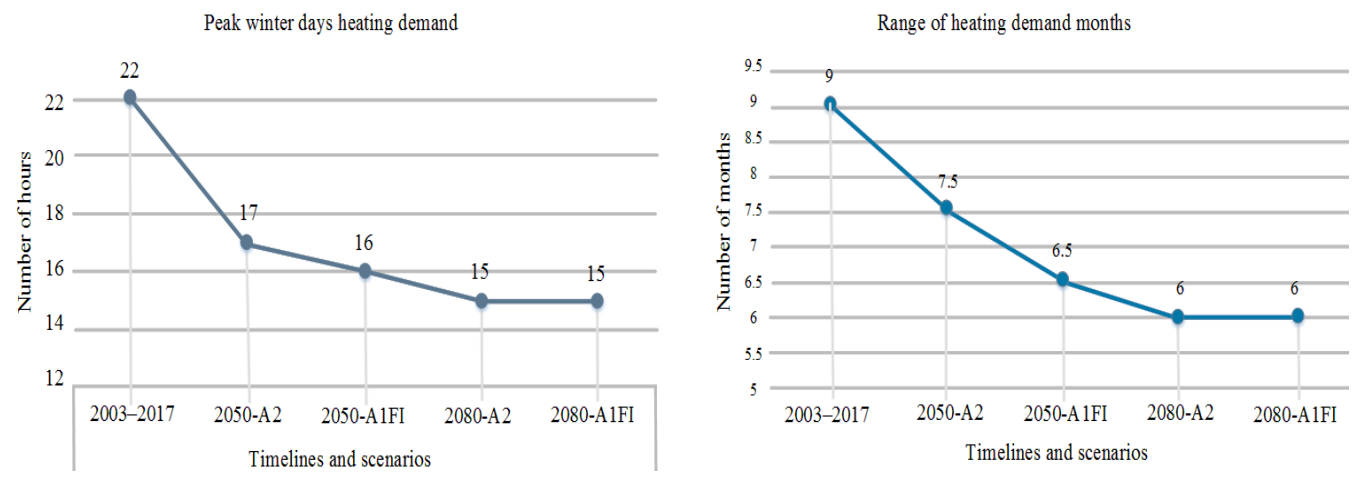

Fig. 9. Heating Period Specific Comparison.

\subsection{Psychrometry}

Psychrometry showed how the climate change impact is pushing the thermal status of the environment to its limits. The thermal comfort zone is a result of a multiple parameters status that decides the $5-6{ }^{\circ} \mathrm{C}$ range placement on the chart, moreover, the previous comparison in Fig. 6 shows how the outcome values of the equations calculations shifts to higher placements with higher GHG emission scenario. The thermal comfort zone bottom and top limits have been pushed up by $1.1^{\circ} \mathrm{C}$ and $1.3{ }^{\circ} \mathrm{C}$ respectively, proving that the building coping mechanism of the climate for the thermal comfort zone might not be able to catch up and endure the climate change impact with higher GHG emission levels, which is technically not a long time away from the 2020 s at all.

Additionally, the passive methods are theoretically proven to take the same effect as the thermal comfort zone, through their placements on the psychrometric chart, with passive cooling limit being pushed up and further through the effective ceiling of operability. 
Furthermore, natural ventilation might not be as effective in the $2080 \mathrm{~s}$ as it is currently, due to higher GHG emissions.

\section{CONCLUSION}

In accordance to the outcome form this research, simulations have shown that climate change will influence the way the current buildings will operate in the future in a negative way, where the thermal specifications and calculations depending only on the current weather data will vary in the future, creating a weather-related performance gap.

In terms of achieving the aim of the research, the methodological process has been found useful for providing a precaution strategy throughout the building design process stages, especially for the pre-design analysis and during design simulations, as the use of the methodology has provided a comprehensive description of the future behaviour of the thermal status, making the aimed at performance have a longer lifespan, that is predicted to endure future weather scenarios of different GHG emission intensities, if dealt with properly.

As the simulation results stands, cooling and heating periods are predicted to vary in range and intensity, leading to a contrast in thermal loads values while going further in time towards the future higher climate change impacts, therefore, the process has provided a future-flexible approach that could give the opportunity of accounting for weather variations, leading to more controllable options for buildings in the future, in terms of retrofitting, heating and cooling demands expectations and energy consumption, where these terms are strongly related to psychrometry and thermal description. Relatively, designers of low-energy building concepts calculate the building performance aspects in respect of high efficiency components behaviour to the climate, where the U-values and building physics plans might differ in operation during climate change effects take place, due to the disparity between results of current weather-dependable simulations and future weather variations of climate change.

In other words, passive methods might be affected in a negative way if their operability was measured only according to the recent weather data only, as seen from the psychrometry analysis, where natural ventilation is not expected be working as effective as it has been designed to be at certain times in the near future. Same expectations for passive solar heating, as it might produce higher temperatures while in effect, changing the purpose they have been designed for, letting alone the thermal comfort zone that could come in discrepancy with the passive methods, where deficiency is predicted to happen.

Eventually, the implications of passive methods rely on producing a platform that could set a base for mitigating climate change impacts on building performance in the future, by implementing precautious measures to prepare the building for future climate scenarios.

\section{REFERENCES}

[1] Frank T. H. Climate change impacts on building heating and cooling energy demand in Switzerland. Energy and Buildings 2005:37(11):1175-1185. https://doi.org/10.1016/j.enbuild.2005.06.019

[2] World Meteorological Organisation. [Online]. [Accessed 17.02.2020]. Available: https://public.wmo.int/en

[3] Olonscheck M., Holsten A., Kropp J. P. Heating and cooling energy demand and related emissions of the German residential building stock under climate change. Energy Policy 2011:39(9):4795-4806. https://doi.org/10.1016/j.enpol.2011.06.041

[4] IPCC Special Report on Emissions Scenarios (SRES): Summary for policymakers - A special report of IPCC working group III intergovernmental panel on climate change. Geneva, Switzerland: IPCC, 2000. 
[5] Chow D. H. C., Levermore G. New algorithm for generating hourly temperature values using daily maximum, minimum and average values from climate models. Building Services Engineering Research and Technology 2007:28:3:237-248. https://doi.org/10.1177/0143624407078642

[6] Belcher S. E., Hacker J. N., Powell D. S. Constructing design weather data for future climates. Building Services Engineering Research and Technology 2005:26(1):49-61. https://doi.org/10.1191/0143624405bt112oa

[7] Jentsch M. F., Bahaj A. S., James P. A. B. Climate change future proofing of buildings - Generation and assessment of building simulation weather files. Energy and Buildings 2008:40(12):2148-2168. https://doi.org/10.1016/j.enbuild.2008.06.005

[8] Jentsch M. F., et al. Transforming existing weather data for worldwide locations to enable energy and building performance simulation under future climates. Renewable Energy 2013:55: 514-524.

https://doi.org/10.1016/j.renene.2012.12.049

[9] Sabunas A., Kanapickas A. Estimation of climate change impact on energy consumption in a residential building in Kaunas, Lithuania, using HEED software. Energy Procedia 2017:128:92-99.

https://doi.org/10.1016/j.egypro.2017.09.020

[10] Arima Y., et al. Effect of climate change on building cooling loads in Tokyo in the summers of the 2030s using dynamically downscaled GCM data. Energy and Buildings 2016:114:123-129. https://doi.org/10.1016/j.enbuild.2015.08.019

[11] Kikumoto H., et al. Study on the future weather data considering the global and local climate change for building energy simulation. Sustainable Cities and Society 2014:14:404-413. https://doi.org/10.1016/j.scs.2014.08.007

[12] Wan K. K. W., et al. Impact of climate change on building energy use in different climate zones and mitigation and adaptation implications. Applied Energy 2012:97:274-282. https://doi.org/10.1016/j.apenergy.2011.11.048

[13] Zhu M., et al. An alternative method to predict future weather data for building energy demand simulation under global climate change. Energy and Buildings 2016:113:74-86. https://doi.org/10.1016/j.enbuild.2015.12.020

[14] Huang J., Gurney K. R. The variation of climate change impact on building energy consumption to building type and spatiotemporal scale. Energy 2016:111:137-153. https://doi.org/10.1016/j.energy.2016.05.118

[15] The World Climate Research Programme (2019). The World Climate Research Programme's (WCRP's) coupled model intercomparison project phase 3 (CMIP3) multi-model dataset. [Online]. [Accessed 17.02.2020]. Available: https://gdo.dcp.ucllnl.org/downscaledemip_projections/n.d

[16] Wang X., et al. Assessment of climate change impact on residential building heating and cooling energy requirement in Australia. Building and Environment 2010:45(7):1663-1682. https://doi.org/10.1016/j.buildenv.2010.01.022

[17] Jiang A., et al. Hourly weather data projection due to climate change for impact assessment on building and infrastructure. Sustainable Cities and Society 2019:50:101688. https://doi.org/10.1016/j.scs.2019.101688

[18] University of North Florida, Department of Construction Management. Weather Morph: Climate Change Weather File Generator. [Online]. [Accessed 17.02.2020]. Available: http://139.62.210.131/weatherGen/

[19] Swiss Federal Office of Meteorology and Climatology. [Online]. [Accessed 17.02.2020]. Available: https://www.meteoswiss.admin.ch/home/ climate/climate-change-in-switzerland/climate-change-scenarios.html

[20] Takashi T. S., et al. MIROC4h-A new high-resolution atmosphere-ocean coupled general circulation model. Journal of the Meteorological Society of Japan 2012:90:3:325-359. https://doi.org/10.2151/jmsj.2012-301

[21] Intergovernmental Panel on Climate Change (IPCC). [Online]. [Accessed 20.08.2020]. Available: https://www.ipcc.ch/data/

[22] Jimenez-Bescos C., Oregi X. Implementing User Behaviour on Dynamic Building Simulations for Energy Consumption. Environmental and Climate Technologies 2019:23(3):308-318. https://doi.org/10.2478/rtuect-20190097

[23] Albatayneh A., et al. The Significance of Building Design for the Climate. Environmental and Climate Technologies 2018:22(1):165-178. https://doi.org/10.2478/rtuect-2018-0011

[24] Rucevskissandris S., Akishin P., Korjakins A. Performance Evaluation of an Active PCM Thermal Energy Storage System for Space Cooling in Residential Buildings. Environmental and Climate Technologies 2019:23(2):165-178. https://doi.org/10.2478/rtuect-2019-0056

[25] Szokolay S. V. Heat: the thermal environment, Introduction to Architectural Science: The Basis of Sustainable Design. Second edition. Linacre House, Oxford, 2008.

[26] Olgyay V. Design with Climate: Bioclimatic Approach to Architectural Regionalism. Princeton University Press, 2015.

[27] Ogunsote O. O., Prucnal-Ogunsote, B. Comfort Limits for the Effective Temperature Index in the Tropics: A Nigerian Case Study. Architectural Science Review 2002:45(2):125-132. https://doi.org/10.1080/00038628.2002.9697500

[28] Choudhury P. K., et al. Datta, Improving Thermal Comfort in Clothing, Woodhead Publishing Series in Textiles, 2011.

[29] NASA GLOBAL Climate Change: Vital Signs of the Planet., Arctic Sea Ice Minimum. [Online]. [Accessed 22.02.2020]. Available: https://climate.nasa.gov/vital-signs/arctic-sea-ice/ 